\title{
Fundamental research in heaping, mixing, and segregation of granular materials: challenges and perspectives
}

\author{
J.M. Ottino ${ }^{\mathrm{a}, *}$, D.V. Khakhar ${ }^{\mathrm{b}}$ \\ a Departments of Chemical and Mechanical Engineering, R.R. McCormick School of Engineering and Applied Science, Northwestern University, \\ 2145 Sheridan Road, Evanston, IL 60208-3120, USA \\ ${ }^{\mathrm{b}}$ Department of Chemical Engineering, Indian Institute of Technology-Bombay Powai, Bombay 400076, India
}

\begin{abstract}
Granular materials are in vogue; the situation is radically different than that of a decade ago. In fact, it can be argued that many of the necessary building blocks for understanding of industrial systems are already here. However, there is still a mismatch between basic research and needs. This is not likely to be bridged: Basic research is divergent and long-term, whereas technological needs are convergent and tied to deadlines. It is however apparent that theoretical understanding is now considerably deeper, that involved simulations are now possible, and that new experimental techniques are opening new horizons. The assembly of all these methodologies, in due time, should open the mixing arts to serious engineering analysis and predictability.
\end{abstract}

Keywords: Granular materials; Heaping; Mixing; Segregation

\section{The academic fascination with granular matter}

Granular materials are in vogue. A few numbers from the Institute of Scientific Information are revealing. Consider the number of papers with the key words granular matter, granular material, and granular materials. The numbers for the period 1982-1988 are 1, 27, and 93; the corresponding numbers for the period 1995-2001-the 2001 period, at the moment of this writing, being woefully incomplete-are 40, 593, and 795 (the numbers for the older terms particulate systems and particle systems are $24 / 181$ and $115 / 609)$.

Why the fascination? Was not it only a few years ago that academic research in this area was deemed to be in a state of crisis [1]? Why the explosion of research in the physics of granular matter? Why does nearly every issue of Physical Review Letters seem to contain a paper in this area? It may useful to list the motivating factors driving academic researchers.

The factors are many and they are all interconnected. The first is that there is new physics and that open theoretical questions abound [2]; the underlying issue is the recognition of granular materials as a new state of matter
[3]. This factor has an experimental counterpart. Experimentation in this area, although it often requires far more sophistication than may appear at first glance, is still accessible and creativity plays a more important role than sheer scale of instrumentation. Another element is that intuition built on fluids often does not work; this represents a challenge. Also, as opposed to Newtonian homogeneous fluids, there is not a clear starting point for analysis; in fact, the methodology depends on the issue at hand. A final element for the appeal-one that undoubtedly should resonate with the readers of this journal-is the clear interplay between science (understanding and explaining) and technology (making and building); it is apparent that inroads into sophisticated scientific questions can have an immediate impact on technology and practice.

\section{Where does the knowledge reside?}

The literature on granular flows is scattered among various branches of engineering-chemical, civil, and mechanical-as well as geophysics, pharmacy, materials science, and powder metallurgy. The most decidedly science-based work appears in physics. The area is attracting first-rank talent. For example, the banner of granular matter research in France is being carried by Pierre de Gennes (Nobel Prize Physics 1991). 
Fascinating work is being carried out. The work spans the spectrum from dry materials to slurries and progress is being made on all fronts (see for example Ref. [4]). The popular imagination is being captured as well [5]. But this may not be fast enough for industrial needs. There are two main obstacles. The first is the sheer range of specific needs. The dominant issues are industry dependent. In pharmaceuticals, it is quality assurance whereas in consumer products - understood as anything from foodstuffs to detergents-it is customization. Cosmetics, powder metallurgy, abrasives, manufacture of solid rocket propellants, solid state combustion, and ceramics all use granular materials but the dominant issues make them look all different [6,7]. The second obstacle is time scales. The objective in industry is to solve problems now, understanding playing a secondary role. Thus, an acceptable solution may be arrived at by massive experimentation or by a clever series of statistically designed experiments. The approach has been successful in many fronts (e.g. granulation, compaction, calcination in rotary kilns, mixing of pharmaceutical powders). But it is clear that the knowledge so gained is of different kind than through a firstprinciples approach. Specific solutions are rarely extensible; scale up is difficult [8].

In theory, a science-driven approach with the long-range goal of producing principle-based knowledge is the solution. However, the systems studied so far are simple (for example, rotating cylinders, quasi-2D heaps), far simpler than the typical problems found in industry. The "real world" is 3D, particles multi-dispersed, sticky, humid or partially wet, the shapes unwieldy. Academic particles are spheres, size distributions are controlled (unimodal or bimodal), geometries are simple, often 2D. But it would be unwise to dismiss these developments as having little relevance to industrial application.

\section{Pouring and tumbling; granular flow}

Let us consider two problems that have attracted significant recent attention: formation of heaps [10,11] and mixing by tumbling [9] (Fig. 1). We use them to illustrate issues that have to do with general aspects of granular flows. There is a clear link between the two problems: both are dominated by surface flows (these may be continuous or in the form of avalanches). In both cases, a layer of particles flow due to gravity while the remaining particles form a fixed bed, on which the flow occurs. Particles in the layer may be absorbed by the bed and vice versa depending on the local conditions. In the case of tumbling, the interchange rate is easily obtained from the angular velocity of the fixed bed, since the interface is fixed. The situation is more complex in the case of heaps (here $\beta$ is the local angle of the interface between the flowing layer and the non-moving bed). Experiments and theory indicate that the interchange flow between the layer and the bed is largely governed by the local surface angle $[12,13]$ : if $\beta>\beta_{\mathrm{m}}$ (where $\beta_{\mathrm{m}}$ is an angle of friction characteristic of the material and the local average velocity), the flow is from the flowing layer to the bed and the interchange is reversed if $\beta<\beta_{\mathrm{m}}$.

A few of the central issues pertaining to shear flow in the layer can be understood in terms of simple scaling arguments. The most obvious analogy of flow of granular matter is a liquid or a gas. The issues are considerably different though [3]. Consider a typical grain with mass $m$, diameter $d=100 \mu \mathrm{m}$, and speed $1 \mathrm{~cm} / \mathrm{s}$. Its kinetic energy is $10^{-12} \mathrm{~J}$, the same order of magnitude of the potential energy $m g d$. By contrast, the necessary temperature to achieve a comparable value of $\kappa T$ is $10^{11} \mathrm{~K}$ [4]! Brownian diffusion is unimportant but particle collisions generate motions that are analogous to thermal motions. The dominance of gravity forces results in high bulk densities in the flowing layer which give rise to high energy dissipation due to interparticle collisions and friction $[14,15]$. Assuming that the local viscous dissipation ( $\tau \dot{\gamma}$, where $\dot{\gamma}$ is the shear rate and $\tau=\rho g d \sin \beta$ is the gravitational shear stress) is balanced by the rate of dissipation $\left(\rho d^{2} \dot{\gamma}^{3}\right)$ the shear is a constant $(\dot{\gamma} \sim(g \sin$ $\left.\beta / d^{1 / 2}\right)$ ) [13]. These results are substantially verified by heap flow experiments, which appear to be a good means of probing the fundamentals of granular flows [12].

\section{Modeling}

Heaping and mixing problems can be modeled; approaches can be divided into discrete and continuum. Discrete approaches encompass Particle Dynamics (PD), Monte Carlo (MC), and Cellular Automata calculations (CA) [16-18]. MC simulations are often too idealized to mimic specific materials; CA computations often yield

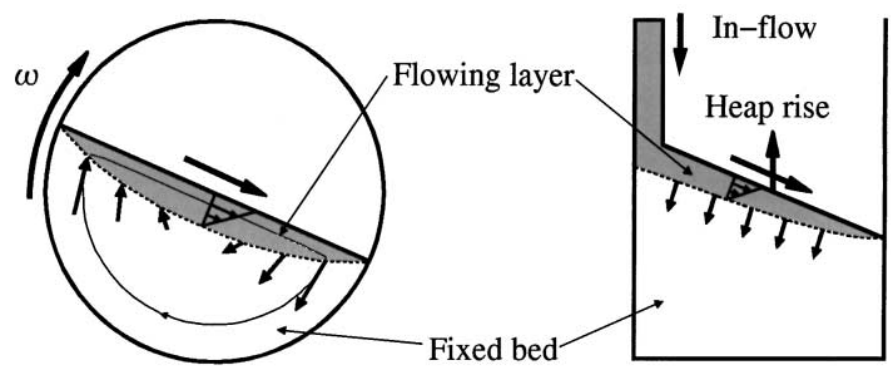

Fig. 1. Surface flows in tumbling and in heap formation. 
considerable insight but at the cost of sacrificing specificity. PD methods come close to the ideal of a first-principles approach. The technique is based on the methodology of molecular dynamics developed for the study of liquids and gases, but, as we shall see, with some important differences. In the simplest case when the grains are large (say $100 \mu \mathrm{m}$ or more), the interactions are purely mechanical. The motion of the particles is governed by Newton's laws; the purpose of PD simulations is to compute the evolution of linear and angular momentum of every particle by using appropriate contact force models [19]. Thus, as opposed to conventional molecular dynamics, torques are transmitted. Dissipation plays an important role.

Though in principle 'exact', PD simulations require precise physical properties (Young's moduli, restitution coefficients, Poisson ratios, etc.) [20]. Predictive calculations for specific materials with complex shapes and multi-dispersed sizes are, in general, nearly impossible. The number of particles is limited to about $10^{4}$ [21], in contrast to practical systems which have $\gg 10^{9}$ particles. However, notwithstanding questions about the nature of the contact force models-even in the case of spheres many important questions remain - there are several drawbacks with this approach. First is that the result of a single simulation is as specific as a single experiment-prediction is gained but understanding is not assured. There is another aspect as well. This has to do with complexity, this term understood in a technical sense. Granular material may be one of the simplest prototypes of complex systems [22].

First principles-based continuum models have been arrived at using analogues of the kinetic theory of hard spheres for nearly elastic spheres [23]. Simplified models for surface flows are obtained by averaging across the layer and using empirical constitutive equations [24]. The main obstacle in continuum descriptions is the importance of intermediate scales (mesoscales). Manifestations occur in jamming and stress chains $[25,26]$. Nevertheless, there is emerging evidence that simplified continuum-based descriptions, with constitutive relations supported by particle dynamics simulations, may form the basis of a general expandable and coherent framework for the description of a variety of flow and segregation processes of granular materials [9]. An advantage of continuum descriptions is that they allow for a clear connection with non-linear based studies and investigation of complex dynamics. The systems considered are (on first viewing) simple; however, their simplicity allows the identification of basic features that had been previously lost in the complexity of the physics or the complexity of the flows.

\section{Complexity}

Science rests on the assumption that understanding of building blocks allows the understanding of the entire system. This is the so-called reductionist approach, i.e. building understanding up from well-understood building blocks. This viewpoint has been remarkably successful since the time of Galileo. It is becoming clear, however, that there are limits to this approach and that complementary viewpoints are needed. There are many processes and systems where interaction among the elementary building blocks - no matter how well-understood-does not even give a glimpse of the behavior of the global system itself. These types of systems are referred to as complex. Complex systems need an integrationist approach.

Consider the case of granular matter. It may be argued that there are no surprises at the level of particles (the particles would be called agents in the complex systems literature). However, when the particles are many and the material is vibrated or tumbled surprising behavior emerges. Materials display structure without any external organizing principle being applied [27]. They self-organize as a consequence of synthesis and collective phenomena, and the behavior cannot be understood in terms of the systems constitutive elements alone. Modeling work in this area (CA) is based on the assumption that by sacrificing accuracy (that is, by painting a cruder caricature of the building blocks) and by focusing on interactions, one may obtain more generality and understanding of systems as a whole. This aspect-common to many dissimilar systems across a variety of disciplines-is a driver of academic research in the self-organization area (for recent reviews the reader may consult the March 7 issue of Nature, pp. 241-284).

\section{Mixing and segregation}

Particularly intractable are problems involving segregation. Segregation is an inescapable byproduct of flow. Segregation negates the effects of mixing - to the point that one may find cases of optimal mixing times. For example, in tumbling, there is an optimal number of revolutions-beyond this point mixing gets worse. Pouring material creates stratification (Fig. 2): for example, when material is poured between two parallel walls (a 2D heap) a highly regular pattern with streaks of uniform thickness is formed. Makse et al. [28] used a CA model to explain these results. In the model, rectangular particles (of different heights but the same width) are added one at a time close to one edge. A particle "rolls" along the surface and comes to rest (always vertical) when the local slope is less than the maximum static angle of repose. If a particle "rolls" to the edge of the heap, the heap is said to be unstable and all particles "roll" until the local slope at each point on the surface is less than the minimum static angle of repose. Thus, a difference in the angles of friction of the particles is sufficient to generate the segregated pattern. Another example of self-organization is provided by the competition between mixing, which often creates a 


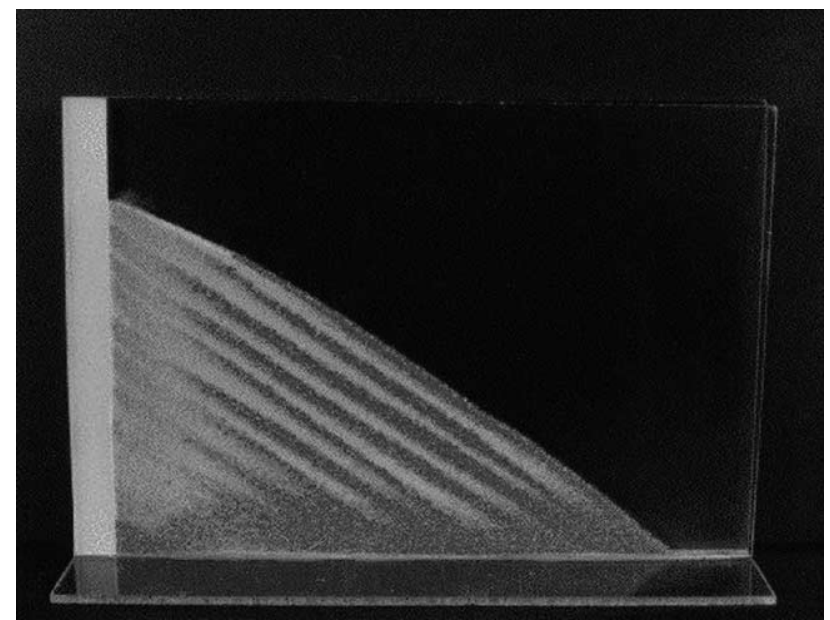

Fig. 2. Stratification of materials of different sizes in the formation of a heap confined between two plates [28]. Reproduced with permission from Nature.

chaotic environment, and segregation. Granular mixtures of just barely different materials segregate according to density and size and the rich array of behaviors is ideally suited for nonlinear-dynamics-based inspection. Consider the example shown in Fig. 3 [29]. There is a dynamic equilibrium between mixing and size-driven segregation resulting in the structure shown in the figure. A slight increase in the degree of filling makes the structure disappear [29].

The effects of segregation can be understood in terms of drift velocities with respect to the mean mass velocity; this has been attempted for $D$-systems (particles differing in



Fig. 3. Pattern formation resulting from a competition between size segregation and mixing in a rotating container in the continuous flow regime [29]. Reproduced with permission from Proc. Natl. Acad. Sci. U. S. A. density) [17]; the case of $S$-systems (particles differing in size) remains largely unexplored. Kinetic theories for hard sphere mixtures provide guidance in this development [30,31]. Consider for example the interplay between chaos and organization in granular flows. The effects of segregation can be understood in terms of drift velocities with respect to the mean mass velocity. Adding this feature into the model results in an important change in the mathematical structure of the system. The system becomes dissipative, and particle trajectories of a component in the mixture converge to an attractor for that component in the absence of diffusion [29]. Experiments reveal that one class of particles moves towards the regular regions, the other towards the chaotic regions [29].

\section{Future and open problems}

Research is likely to be divergent, whereas technology needs to be convergent (Fig. 4). Research is also disorganized; there is always overshooting; initial claims are invariably too optimistic. Consider, for example, pattern formation in thin shaken layers. The direct industrial relevance is thin: the amplitudes and frequencies are way out of bounds to those relevant to typical industrial practice. Similar criticisms may apply to work on avalanches-there is little obvious industrial relevance, the work on 2D flows being too idealized. But is hard to take shortcuts when building knowledge; understanding is built one step at a time. One cannot climb a ladder without the first steps being firmly in place. Also one should be wary of focusing things too much. The inevitable consequence of focusing too early on the convergent is that potential new ideas are eliminated. It is more instructive to list open problems that may be relevant to both industry and academia.

Many of the necessary building blocks for understanding of industrial systems are already here. Scouting can yield significant results for those who are persistent enough and have the right training (or work hard at acquiring one). Thus, for example, existing building blocks allow the design of kilns (divergent process, Fig. 4). Consider a rotating cylinder for continuous coating of particles where
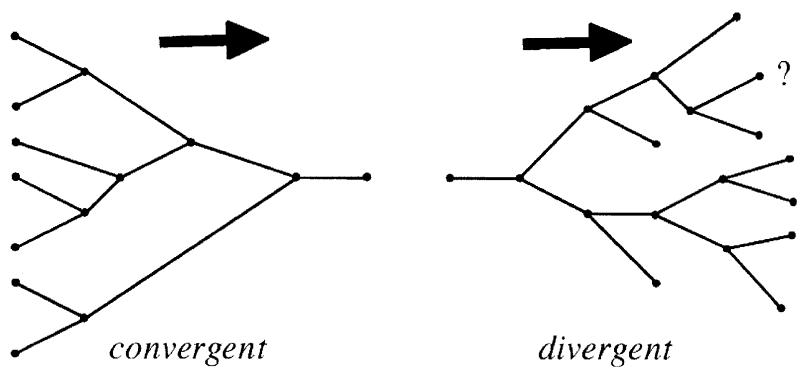

divergent

Fig. 4. In convergent work, various elements contribute to the solution of a problem; in divergent work, one central idea acts as a generating point and considerably branching leading to the creation and potential solution of many diverse problems. 
the free surface is sprayed by solvent with the coating material dissolved in. The frequency of exposure of fresh material and the rate of cross-sectional mixing are important for uniformity of coating. 2D experiments with different arrangements of baffles are useful for evaluating these aspects. The residence time and axial dispersion produce a distribution of coating thicknesses. Both these can be estimated from theory and reported data [32,33]. Similar comments apply to scale-up. On-going fundamental work is uncovering the effect of change of scale in surface flows.

Another aspect of fundamental work is to open new avenues (divergent process, Fig. 4). Consider the discovery of chaos in granular flows. The most direct application appears to be tumbling mixers, though applications to high shear mixers (plough share) and convective mixers (rotating screw) appear possible as well. An important aspectwhich surprisingly may make granular studies simpler that those of fluids - is that the flow of granular materials in most mixers is characterized by relatively small regions of granular motion while most of the particles are in nearly fixed beds. This is very different from the case of fluids and should aid in determining mixer design and suggesting new configurations.

New experimental techniques are also opening new horizons. It is now possible to quantitatively measure velocities in opaque granular media [25,34-36]. More work should go into this area. However, what is not sufficiently developed is the ability to infer velocity fields from digitized data. Resolution of measurements using non-invasive techniques is not good, particularly for estimating volume fractions. This is especially important since small changes in solids volume fractions translate to large stress variations in dense flows. An alternate approach is to write stress constitutive equations in terms of macroscopic variables in specific flows (for example, an effective friction coefficient that depends on the height and average velocity in a surface flow). Thus, generality is lost but specific practical problems could be tackled. Rheometers for determining such material functions are not available. New techniques, such as particle tracking velocimetry (PTV), are starting to provide vast quantitative data to characterize granular flows [37].

Segregation is imperfectly understood and is another area where more work is needed. Experimentally, the most studied systems are S-systems; whereas theoretically, the most studied systems are $D$-systems. Very little has been done on either front on the effects of particle shape. One of the end-results in this area is to obtain constitutive relations that can be coupled in continuum-based models. Discretized models should be explored as well. There are just a handful of agent-based models of mixing and segregation. On the purely PD-modeling side, there is a dearth of cross comparisons (as for example there have been for visco-elastic fluid flows) for simulation methodologies involving different contact force models. This would proba- bly require adopting a specific problem as the standard (as for example was the 2:1 contraction in visco-elastic fluid mechanics).

It is apparent that the assembly of all these methods, in due time, should open the mixing arts to serious engineering analysis and predictability.

\section{References}

[1] B.J. Ennis, J. Green, R. Davis, Legacy of neglect in the United States, Chem. Eng. Prog. 90 (1994) 32-43.

[2] P.G. de Gennes, Granular matter: a tentative view, Rev. Mod. Phys. 71 (1999) S374-S382, Sp. Iss.

[3] H.M. Jaeger, S.R. Nagel, R.P. Behringer, Granular solids, liquids, and glasses, Rev. Mod. Phys. 68 (1996) 1259-1273.

[4] J. Duran, Sands, powders and grains. An Introduction to the Physics of Granular Materials, Springer, New York, 2000.

[5] P. Ball, The Self-made Tapestry: Pattern Formation in Nature, Oxford Univ. Press, Oxford, 1999.

[6] J. Van Cleef, Powder technology, Am. Sci. 79 (1991) 304-315.

[7] N. Harnby, An engineering view of pharmaceutical mixing, Pharm. Sci. Technol. Today 3 (2000) 303-309.

[8] L.T. Fan, Y.-M. Chen, F.S. Lai, Recent developments in solids mixing, Powder Technol. 61 (1990) 255-287.

[9] J.M. Ottino, D.V. Khakhar, Mixing and segregation of granular materials, Ann. Rev. Fluid Mech. 32 (2000) 55-91.

[10] J.P. Bouchaud, M. Cates, J. Ravi Prakash, S. Edwards, A model for the dynamics of sandpile surface, J. Phys. I (France) 4 (1994) 1383-1409.

[11] T. Boutreux, P.G. deGennes, Surface flows of granular mixtures: I. General principles and minimal model, J. Phys. I (France) 6 (1996) 1295-1304.

[12] D.V. Khakhar, A.V. Orpe, P. Andresén, J.M. Ottino, Surface flow of granular materials: heap formation, J. Fluid Mech. (2001) in press.

[13] S. Douady, B. Andreotti, A. Daerr, On granular surface flow equations, Eur. Phys. J. B 11 (1999) 131-142.

[14] P. Coussot, C. Ancey, Rheophysical classification of concentrated suspensions and granular pastes, Phys. Rev. E 59 (1999) 4445-4457.

[15] J. Rajchenbach, in: H. Hermann (Ed.), Physics of Dry Granular Media, Kluwer Academic, Netherlands, 1998, p. 421.

[16] P.A. Cundall, D.L. Strack et al., A discrete numerical model for granular assemblies, Geotechnique 29 (1979) 47-65.

[17] D.V. Khakhar, J.J. McCarthy, J.M. Ottino, Radial segregation of materials in rotating cylinders, Phys. Fluids 9 (1997) 3600-3614.

[18] P. Bak, C. Tang, K. Wiesenfeld, Self-organized criticality: an explanation of the 1/f noise, Phys. Rev. Lett. 59 (1987) 381-384.

[19] J. Schafer, S. Dippel, D.E. Wolf, Force schemes in simulation of granular materials, J. Phys. I 6 (1996) 5-20.

[20] P.W. Cleary, G. Metcalfe, K. Liffman, How well do discrete element granular flow models capture the essentials of mixing processes? Appl. Math. Model. 22 (1998) 995-1008.

[21] T. Shinbrot, F.J. Muzzio, Nonequilibrium patterns in granular mixing and segregation, Phys. Today 53 (2000) 25-30.

[22] N. Goldenfeld, L.P. Kadanoff, Simple lessons from complexity, Science 284 (1999) 87-89.

[23] C.K.K. Lun, S.B. Savage, D.J. Jeffrey, N. Chepurniy, Kinetic theories for granular flow: inelastic particles in couette flow and slightly inelastic particles in a general flow field, J. Fluid Mech. 140 (1984) 223-256.

[24] S.B. Savage, K. Hutter, The motion of a finite mass of granular material down a rough incline, J. Fluid Mech. 199 (1989) 177-215.

[25] C.S. O’Hern, S.A. Langer, A.J. Liu, S.R. Nagel, Force distributions near the jamming and glass transitions, Phys. Rev. Lett. 86 (2001) $111-114$. 
[26] D.W. Howell, R.P. Behringer, V.J. Veje, Fluctuations in granular media, Chaos 9 (1999) 559-572.

[27] P.B. Umbanhowar, P.B. Melo, H.L. Swinney, Localized excitations in a vertically vibrated granular layer, Nature 382 (1996) 793-796.

[28] H.A. Makse, S. Havlin, P.R. King, H.E. Stanley, Spontaneous stratification in granular mixtures, Nature 386 (1997) 379-381.

[29] K.M. Hill, D.V. Khakhar, J.F. Gilchrist, J.J. McCarthy, J.M. Ottino, Segregation-driven organization in chaotic granular flows, Proc. Natl. Acad. Sci. U. S. A. 96 (1999) 11701-11706.

[30] J.T. Jenkins, F. Mancini, Kinetic theory for binary mixtures of smooth, nearly elastic spheres, Phys. Fluids A 1 (1989) 2050-2057.

[31] D.V. Khakhar, J.J. McCarthy, J.M. Ottino, Mixing and segregation of granular materials in chute flows, Chaos 9 (1999) 594-610.

[32] M. Hehl, H. Kroger, H. Helmrich, K. Schugerl, Longitudinal mixing in horizontal drum reactors, Powder Technol. 20 (1978) 29-37.
[33] S.J. Rao, S.K. Bhatia, D.V. Khakhar, Axial transport of granular solids in rotating cylinders: Part 2. Experiments in a non-flow system, Powder Technol. 67 (1991) 153-162.

[34] M. Nakagawa, S.A. Altobelli, A. Caprihan, E. Fukushima, E.K. Jeong, Non-invasive flows of granular materials by magnetic resonance imaging, Exp. Fluids 16 (1993) 54-60.

[35] N. Menon, D.J. Durian, Diffusing-wave spectroscopy of dynamics in a three-dimensional granular flow, Science 275 (1997) 1920-1922.

[36] B.F.C. Laurent, J. Bridgwater, D.J. Parker, Motion in a particle bed agitated by a single bed, AIChE J. 46 (2000) 1723-1734.

[37] N. Jain, R.M. Lueptow, J.M. Ottino, An experimental of the flowing granular layer in a rotating tumbler, Phys. Fluids (2001) submitted. 